Gynäkologe 2010 • 43:551-551

DOI 10.1007/s00129-010-2534-4

Online publiziert: 1. Juli 2010

๑) Springer-Verlag 2010

\author{
W. Distler ${ }^{1} \cdot$ G. Emons ${ }^{2} \cdot$ N. Maass ${ }^{3}$ \\ ${ }^{1}$ Klinik und Poliklinik für Frauenheilkunde und Geburtshilfe, \\ Universitätsklinikum Carl Gustav Carus, Dresden \\ ${ }^{2}$ Klinik für Gynäkologie und Geburtshilfe, Universitätsmedizin \\ Göttingen, Georg-August-Universität, Göttingen \\ ${ }^{3}$ Frauenklinik für Gynäkologie und Geburtshilfe, \\ Universitätsklinikum der RWTH, Aachen
}

\section{Molekularbiologische Mechanismen für den Kliniker}

und Proliferation der Körperzelle fördern oder hemmen. Zunehmend treten Ärzte und Naturwissenschaftler in einen Dialog, um die Diagnostik, Behandlung und Prävention, insbesondere von Krebserkrankungen, gemeinsam zu diskutieren. Es ist schon jetzt klar, dass in den nächsten 10 Jahren buchstäblich hunderte neuer biologischer und/oder pharmakologischer Substanzen gegen spezifische, molekulare Intermediärprodukte der Krebszelle erprobt und dann auch mit therapeutischem Ansatz eingesetzt werden. Dabei sollte der verantwortungsvolle Kliniker immer einerseits wissen, welche molekularen Mechanismen das Leiden verursachen, und andererseits, welche molekularbiologischen Therapeutika mit einem Minimum an Nebenwirkung zum Einsatz kommen können.

\section{( Erprobt werden buchstäblich hunderte neuer Substanzen gegen Intermediärprodukte der Krebszelle}

Das vorliegende Themenheft von „Der Gynäkologe " möchte den klinisch tätigen Ärzten einige Bereiche der „biotechnologischen Revolution" näherbringen und damit helfen, die Neugierde auf neue diagnostische und therapeutische Ansätze zu wecken. In knapper Form sagen die angloamerikanischen Kollegen „from the bench to the bed“, also „von der Werkbank zum Krankenbett", und meinen damit die Verknüpfung von Wissenschaft und Klinik.

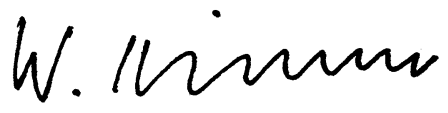

Prof. Dr. W. Distler

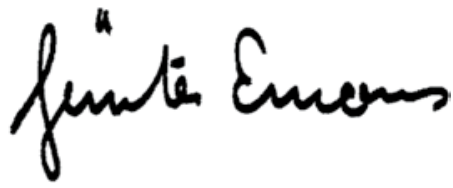

Prof. Dr. G. Emons

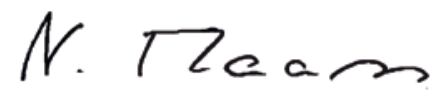

Prof. Dr. N. Maass

\section{Korrespondenzadresse \\ Prof. Dr. W. Distler \\ Klinik und Poliklinik für Frauenheil- kunde und Geburtshilfe, Universitäts- klinikum Carl Gustav Carus, Dresden Fetscherstraße 74, 01307 Dresden frauenklinik@uniklinikum-dresden.de}

Die Molekularbiologie hat unser Verständnis von der Pathogenese vieler erkrankungen, revolutioniert. Wir wissen heute mehr über Molekularmechanismen und genetische Prozesse, die Wachstum Krankheiten, insbesondere der Tumor- 